\title{
Nonperturbative treatments of level shifts of excited states and high-order harmonic generation in strong fields
}

\author{
Shih-I Chu, Kwanghsi Wang, ${ }^{*}$ and Eric Layton \\ Department of Chemistry, University of Kansas, Lawrence, Kansas 66045
}

Received September 1, 1989; accepted November 3, 1989

\begin{abstract}
In this paper we accomplish three goals. First, we present new nonperturbative results of complex quasi-energies (shifts and widths) for several low-lying excited states of atomic $\mathrm{H}$ in strong fields, using the $L^{2}$ non-Hermitian Floquet matrix technique. Second, we present a new nonperturbative $L^{2}$ technique for the treatment of ac Stark shifts of arbitrary excited states. We found that all the Rydberg states in weak fields are upshifted and closely follow the quadratic field dependence described by the ponderomotive potential $e^{2} F^{2} / 4 m \omega^{2}$. Large deviation from the ponderomotive shift and intricate level-shift behaviors, however, occur in strong fields. Finally, we present a classical nonperturbative treatment of the electronic motion in intense laser fields. We show that the spectral analysis of classical trajectories can provide detailed insights regarding the mechanisms responsible for the multiple-harmonic generation recently observed in high-intensity experiments.
\end{abstract}

\section{INTRODUCTION}

Recent experiments on multiphoton ionization (MPI) and above-threshold ionization (ATI) of atoms in strong fields show significant energy shifts and broadenings of ATI peaks. ${ }^{1,2}$ Further, for laser pulse widths of less than 1 psec, the electron energy spectrum can exhibit fine structure in the individual ATI peaks. These observations suggest that the structure of excited states plays a significant role in determining the properties of ATI. This creates the necessity of analyzing the excited-state atomic energy-level structure in the presence of strong fields.

There have been a number of theoretical studies of ac Stark shifts, mostly focused on nonperturbative ${ }^{3-5}$ or highorder perturbative treatments ${ }^{6}$ of the ground state of atomic $H$. Thus our understanding of the properties (including both ac Stark shifts and MPI widths ${ }^{3-5,7}$ ) of the ground state can now be considered rather complete. In contrast, there are only a few perturbative studies on the excited-state properties. ${ }^{8,9}$ In this paper we outline a method for nonperturbative treatment of the ac Stark shifts of atomic $\mathrm{H}$ for any arbitrary excited states. In addition, we present some new nonperturbative results of the ac Stark shifts and MPI total widths (rates) for several low-lying excited states of atomic $\mathrm{H}$, using the $L^{2}$ non-Hermitian Floquet matrix method. ${ }^{10}$

To gain insights regarding the mechanisms responsible for the high-order harmonic generation recently observed in high-intensity MPI/ATI experiments, ${ }^{11-13}$ we have also performed a spectral analysis of the classical trajectories for the electron motion under the influence of both the Coulomb and oscillating electric fields. In addition, to confirm recent quantum-mechanical model predictions, ${ }^{14,15}$ we have identified a general type of classical trajectory responsible for the observed behavior of high-order harmonics generated in intense fields.

In Section 2, we review briefly the $L^{2}$ non-Hermitian Floquet method and present the nonperturbative results of complex quasi-energies for several low-lying states of atomic $H$. In Section 3, we outline a method for the nonperturba- tive treatment of the ac Stark shifts of highly excited states. Finally, the spectral analysis method for classical trajectories is presented in Section 4 along with a discussion of highorder harmonic generation.

\section{INTENSITY-DEPENDENT COMPLEX QUASI-ENERGIES (SHIFTS AND WIDTHS) OF LOW-LYING EXCITED STATES OF ATOMIC HYDROGEN}

The $L^{2}$ non-Hermitian Floquet formulation ${ }^{10,16}$ has recently been extended to the study of intense-field MPI/ATI from the atomic $\mathrm{H}$ ground state. ${ }^{3,4}$ In this section we present new results for several low-lying excited states $(2 s, 2 p, 3 s, 3 p, 3 d)$ of atomic $H$. The method permits nonperturbative and self-consistent treatment of intense-field effects (in that all atomic levels are simultaneously shifted and broadened by the external fields) and straightforward inclusion of freefree transitions and the effects of coupling among electronic continua.

Corresponding to the periodically time-dependent Hamiltonian

$$
\hat{H}(\mathbf{r}, t)=-\left(\hbar^{2} / 2 m\right) \nabla^{2}-e^{2} / r+e F z \cos \omega t
$$

describing the interaction of atomic $\mathrm{H}$ with a monochromatic, linearly polarized, coherent field of frequency $\omega$ and peak field strength $F$, an equivalent time-independent Hamiltonian $\hat{H}_{F}(\mathbf{r})$ may be obtained by an extension of the semiclassical Floquet Hamiltonian method. ${ }^{16-18}$ The structure of $\hat{H}_{F}$ has been documented elsewhere ${ }^{10}$ and is reproduced in Fig. 1 for convenience of discussion. The Floquet Hamiltonian $\hat{H}_{F}$ shows a tridiagonal block structure, consisting of the diagonal $A \pm n \omega I(n=0, \pm 2, \pm 4, \ldots)$ blocks and the off-diagonal $B$ blocks. Each diagonal block is composed of angular momentum blocks $S, P, D, \ldots$, representing the projection of the atomic electronic Hamiltonian onto states of $l=0,1$, $2, \ldots$, and $V_{l, l}$ 's are electric dipole coupling matrix elements. Thus, in the case of atomic $H$, the $S$ block consists of the $1 s$, 


\begin{tabular}{|c|c|c|c|c|c|}
\hline \multirow{5}{*}{$\mathrm{H}_{\mathrm{F}}=$} & $A+4 \omega I$ & B & 0 & 0 & 0 \\
\hline & $B^{T}$ & $A+2 \omega I$ & B & 0 & 0 \\
\hline & 0 & $\mathrm{~B}^{\mathrm{T}}$ & A & B & 0 \\
\hline & 0 & 0 & $B^{T}$ & $A-2 \omega I$ & B \\
\hline & 0 & 0 & 0 & $B^{T .}$ & $A-4 \omega I$ \\
\hline \multicolumn{6}{|l|}{ where } \\
\hline \multirow{5}{*}{$A=$} & $S$ & $\mathrm{~V}_{\mathrm{SP}}$ & 0 & 0 & 0 \\
\hline & $\mathrm{V}_{\mathrm{PS}}$ & $P-\omega I$ & $V_{P D}$ & 0 & 0 \\
\hline & 0 & $V_{D P}$ & D & $V_{D F}$ & 0 \\
\hline & 0 & 0 & $V_{F D}$ & $F-\omega I$ & $V_{P G}$ \\
\hline & 0 & 0 & 0 & $\mathrm{~V}_{\mathrm{GF}}$ & G \\
\hline \multicolumn{6}{|l|}{ and } \\
\hline \multirow{5}{*}{$B=$} & 0 & 0 & 0 & 0 & 0 \\
\hline & $v_{P S}$ & 0 & $V_{P D}$ & 0 & 0 \\
\hline & 0 & 0 & 0 & 0 & 0 \\
\hline & 0 & 0 & $V_{F D}$ & 0 & $V_{F G}$ \\
\hline & 0 & 0 & 0 & 0 & 0 \\
\hline
\end{tabular}

Fig. 1. Structure of the time-independent Floquet Hamiltonian for atomic MPI/ATI.

$2 s, 3 s, \ldots n s, \ldots$ bound states and the entire $k s$ Coulomb continuum. The Hamiltonian of Fig. 1 has no discrete spectrum, and the time evolution is dominated by poles of the resolvent $\left(E-H_{F}\right)^{-1}$ near the real axis but on higher Riemann sheets. These complex poles, which correspond to decaying complex quasi-energy states (QES's), may be found directly from the analytically continued Floquet Hamiltonian, $H_{F}(\alpha)$, obtained by the complex scaling transformation $^{19} \mathbf{r} \rightarrow \mathbf{r} e^{i \alpha}$. This transformation effects an analytical continuation of $\left(E-H_{F}\right)^{-1}$ into the lower half-plane on an appropriate higher Riemann sheet, allowing the complex QES to be determined by solution of a non-Hermitian eigenproblem. The real parts of the complex eigenvalues of $\hat{H}_{F}(\alpha)$ provide the ac Stark shifts, whereas the imaginary parts determine directly the total MPI widths (rates). In practice, the atomic blocks are made discrete by use of a finite subset of the complete Laguerre basis $r^{l+1} e^{-\lambda r} L_{n}{ }^{2 l+2}(\lambda r)$, where $\lambda$ is an adjustable parameter and $n$ $=0,1,2, \ldots$ This yields a Pollaczeck quadrature representation of the bound and continuum contributions to the spectral resolution of the hydrogenic Hamiltonian. In practice, the convergence of MPI calculations may achieve arbitrary precision by systematically increasing the basis size and the number of angular momentum blocks.

Table 1 shows the intensity-dependent complex quasienergies $\left(E_{R},-\Gamma / 2\right)$ of the perturbed low-lying excited states
$(2 s, 2 p, 3 s, 3 p, 3 d)$ of atomic $\mathrm{H}$ at $\lambda=530 \mathrm{~nm}$. The $E_{R}$ 's are the ac Stark-shifted energies, whereas $\Gamma$ 's are the total MPI widths (rates). Up to five Floquet blocks ( $A, A \pm 2 \omega, A \pm$ $4 \omega)$ and $(25 s, 25 p, 25 d, 25 f, 25 g)$ basis functions for each block are used in these calculations to achieve convergence. Strong mixings with some other states have already occurred for each atomic state at the largest $F_{\text {rms }}$ listed. Beyond these field intensities, the quasi-energy eigenvector components are spread among many Floquet states, and the identities of atomic states can no longer be discerned. Figure 2 depicts the intensity-dependent ac Stark behavior of these low-lying excited states. We note that all the low-lying states (except $3 d$ ) are shifted upward as the field intensity increases.

Table 1. Intensity-Dependent Complex Quasi-Energies $^{a}$

\begin{tabular}{|c|c|c|c|}
\hline States & $F_{\mathrm{rms}}$ & $E_{R}$ & $-\Gamma / 2$ \\
\hline \multirow[t]{9}{*}{$2 s$} & $1.0(-4)^{b}$ & -0.12499970 & $-0.1383(-7)$ \\
\hline & $5.0(-4)$ & -0.1249926 & $-0.1853(-7)$ \\
\hline & $1.0(-3)$ & -0.124970 & $-0.8769(-7)$ \\
\hline & $2.0(-3)$ & -0.124876 & $-0.1124(-5)$ \\
\hline & $3.0(-3)$ & -0.124709 & $-0.5125(-5)$ \\
\hline & $4.0(-3)$ & -0.124455 & $-0.1432(-4)$ \\
\hline & $5.0(-3)$ & -0.124102 & $-0.3041(-4)$ \\
\hline & $7.5(-3)$ & -0.12277 & $-0.1092(-3)$ \\
\hline & $1.0(-2)$ & -0.12125 & $-0.5898(-3)$ \\
\hline \multirow[t]{9}{*}{$2 p$} & $1.0(-4)$ & -0.12499905 & $-0.7705(-9)$ \\
\hline & $5.0(-4)$ & -0.1249762 & $-0.5371(-8)$ \\
\hline & $1.0(-3)$ & -0.124905 & $-0.7432(-7)$ \\
\hline & $2.0(-3)$ & -0.124626 & $-0.1159(-5)$ \\
\hline & $3.0(-3)$ & -0.124177 & $-0.5693(-5)$ \\
\hline & $4.0(-3)$ & -0.123581 & $-0.1726(-4)$ \\
\hline & $5.0(-3)$ & -0.122876 & $-0.4047(-4)$ \\
\hline & $7.5(-3)$ & -0.12136 & $-0.6558(-3)$ \\
\hline & $1.0(-2)$ & -0.11898 & $-0.4256(-2)$ \\
\hline \multirow[t]{9}{*}{$3 s$} & $1.0(-4)$ & -0.5555488 & $-0.1846(-6)$ \\
\hline & $5.0(-4)$ & -0.0555383 & $-0.5219(-5)$ \\
\hline & $1.0(-3)$ & -0.055486 & $-0.2096(-4)$ \\
\hline & $2.0(-3)$ & -0.055279 & $-0.8397(-4)$ \\
\hline & $3.0(-3)$ & -0.054933 & $-0.1892(-3)$ \\
\hline & $4.0(-3)$ & -0.054448 & $-0.3370(-3)$ \\
\hline & $5.0(-3)$ & -0.053823 & $-0.5278(-3)$ \\
\hline & $6.0(-3)$ & -0.05306 & $-0.7624(-3)$ \\
\hline & $7.5(-3)$ & -0.05165 & $-0.1197(-2)$ \\
\hline \multirow[t]{8}{*}{$3 p$} & $1.0(-4)$ & -0.05555525 & $-0.2443(-6)$ \\
\hline & $5.0(-4)$ & -0.0555478 & $-0.6329(-5)$ \\
\hline & $1.0(-3)$ & -0.055525 & $-0.2540(-4)$ \\
\hline & $2.0(-3)$ & -0.055432 & $-0.1025(-3)$ \\
\hline & $3.0(-3)$ & -0.055280 & $-0.2341(-3)$ \\
\hline & $4.0(-3)$ & -0.055062 & $-0.4248(-3)$ \\
\hline & $5.0(-3)$ & -0.05479 & $-0.6807(-3)$ \\
\hline & $6.0(-3)$ & -0.05445 & $-0.1010(-2)$ \\
\hline \multirow[t]{7}{*}{$3 d$} & $1.0(-4)$ & -0.05555580 & $-0.1336(-6)$ \\
\hline & $5.0(-4)$ & -0.0555615 & $-0.3369(-5)$ \\
\hline & $1.0(-3)$ & -0.055579 & $-0.1355(-4)$ \\
\hline & $2.0(-3)$ & -0.055642 & $-0.5541(-4)$ \\
\hline & $3.0(-3)$ & -0.055728 & $-0.1288(-3)$ \\
\hline & $4.0(-3)$ & -0.055812 & $-0.2390(-3)$ \\
\hline & $5.0(-3)$ & -0.05587 & $-0.3923(-3)$ \\
\hline
\end{tabular}

${ }^{a}\left(E_{R},-i \Gamma / 2\right)$ (in atomic units) of the perturbed low-lying excited states of atomic $\mathrm{H}$ at $\lambda=530 \mathrm{~nm}$. The rms field strength of $F_{\mathrm{rms}}=1.0 \mathrm{a} . \mathrm{u}$. corresponds to a rms intensity of $7.016 \times 10^{16} \mathrm{~W} / \mathrm{cm}^{2}$.

b $1.0(-4)=1.0 \times 10^{-4}$ 

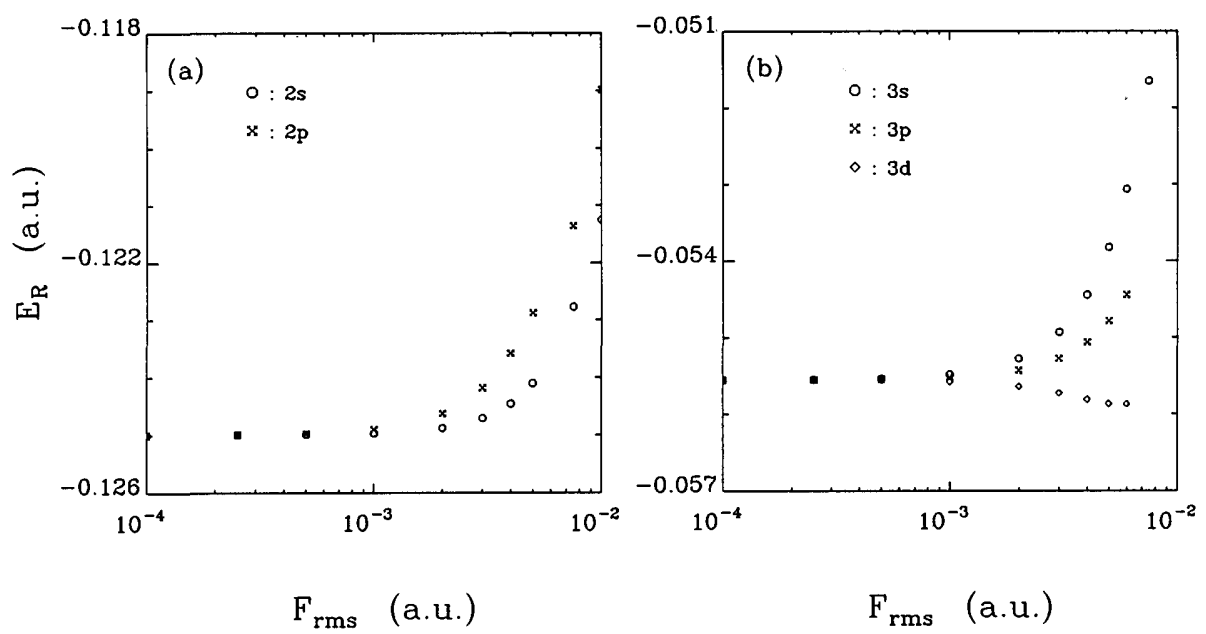

Fig. 2. Intensity-dependent energy-level-shift behavior for low-lying excited states $(2 s, 2 p)$ and $(3 s, 3 p, 3 d)$ of atomic $\mathrm{H}$.

Shifting and splitting of atomic states can be accounted for by perturbation theory for $F_{\text {rms }}<5 \times 10^{-4}$ a.u., as our weakfield results reproduce nearly exactly the perturbative results of Ref. 9. For $F_{\text {rms }} \geq 10^{-3}$ a.u., higher-order effects become significant, and nonperturbative treatment, such as the $L^{2}$ non-Hermitian Floquet calculations presented here, is required to achieve convergence.

\section{ALTERNATING-CURRENT STARK SHIFTS OF HIGHLY EXCITED STATES IN STRONG FIELDS}

The $L^{2}$ non-Hermitian Floquet matrix method described in Section 2 can, in principle, be extended to higher excited states, and the detailed calculations are now in progress. However, it becomes impractical to treat highly excited states, as the Laguerre basis set size needed to achieve convergence increases rapidly as $n$ increases. If one is interested primarily in the energy-level shifts only, one does not need to use complex scaling techniques. Thus, we are starting again from the Floquet Hamiltonian $\hat{H}_{F}$, shown in Fig. 1, except that now it is a Hermitian matrix with only real eigenvalues. If we use the conventional basis expansion method (which usually describes the energy spectrum starting from the ground state), we will have the familiar difficulty-a large number of basis functions are needed to achieve high accuracy, even for intermediate excited states. What we need here is an appropriate $L^{2}$ basis set that can provide a compact, yet accurate, description of any arbitrary excited states without the need for a large number of basis functions. It turns out that the well-known Sturmian basis $\left\{S_{n l}(r)\right\},{ }^{20,21}$ which provides an $L^{2}$ technique for the treatment of both the discrete and continuum properties, is an ideal choice for such a purpose. The gist of using the Sturmian basis is that one can adjust the energy parameters $\alpha$ and $E_{0}$ in the (unperturbed) Coulombic Schrödinger equation (in atomic units)

$$
\left[-1 / 2 \mathrm{~d}^{2} / \mathrm{d} r^{2}+l(l+1) / 2 r^{2}-\alpha / r-E_{0} / 2\right] S_{n l}(r)=0,
$$

where $\alpha=k n=\left(-E_{0}\right)^{1 / 2} n$ such that $E_{0} / 2$ is set equal to some arbitrary highly excited state energy $-1 / 2 n^{2}$. The Sturmian functions $S_{n l}(r)$ thus generated will provide a compact basis for the description of both the discrete states (now centered around some particular (highly excited) states $n$ and the continuum. Table 2 shows an example of how this procedure works. Here we have diagonalized the $s$-states $(l=0)$ atomic $\mathrm{H}$ Hamiltonian

$$
H^{(0)}=-(1 / 2) \mathrm{d}^{2} / \mathrm{d} r^{2}+l(l+1) / 2 r^{2}-1 / r,
$$

using a 40 Sturmian basis function $S_{n l}(r)$, where $n=15$, $16, \ldots 54$ and $l=0$. We have chosen $E_{0}$ in such a way that the Rydberg levels of central interest are $\sim 25$. In fact an energy band of at least 12 excited atomic states $(n=10$ to $n$ $=21$ ) are described accurately to 9 decimal places. The positive-energy pseudodiscrete states describe the continuum. The key feature here is that there is no need to start the basis function expansion from $n=1$ in describing the excited states. The atomic properties can still be converged by treating accurately the excited levels of principal interest and by including a sufficient number of lower-lying bound and pseudocontinuum states in the basis set. The inclusion and accuracy of lower-lying states far from the excited states of interest are of little concern unless there is an accidental photon resonance. The situation here is similar to the conventional $L^{2}$ technique for treating the ground state, where it is known that there is no need to include or treat every excited state correctly provided that the basis set is sufficiently complete. With the appropriately adjusted new Sturmian basis, therefore, we can now construct a more compact and effective Floquet matrix for excited-state problems.

The Sturmian function $S_{n l}(r)$ has the following explicit form:

$$
S_{n l}(r)=N_{n l} e^{-k r}(2 k r)^{l+1} L_{n+l}^{2 l+1}(2 k r)
$$

where $k \equiv\left(-E_{0}\right)^{1 / 2}$, and $L_{n+l} l^{2 l+1}$ is the Laguerre polynomial. We use the convention ${ }^{20}$ that the Sturmians are normalized with respect to weight $2 / r$, namely,

$$
\int_{0}^{\infty} S_{n l}(r)(2 / r) S_{n^{\prime} l^{\prime}}(r) \mathrm{d} r=\delta_{n n^{\prime}} \delta_{l l^{\prime}}
$$

One other advantage of using the Sturmian basis is the 
Table 2. Eigenvalues of Unperturbed Atomic $\mathrm{H}$ ( $s-$ States) Diagonalized in an Extended Sturmian Basis ${ }^{a}$

\begin{tabular}{|c|c|c|c|}
\hline$N$ & $n$ & $E(n)$ Sturmian & $E(n)$ Exact \\
\hline 1 & 15 & -0.008958511 & -0.002222222 \\
\hline 2 & 16 & -0.002675953 & -0.001953125 \\
\hline 3 & 17 & -0.001820747 & -0.001730104 \\
\hline 4 & 18 & -0.001547228 & -0.001543210 \\
\hline 5 & 19 & -0.001385055 & -0.001385041 \\
\hline 6 & 20 & -0.001250000 & -0.001250000 \\
\hline 7 & 21 & -0.001133787 & -0.001133787 \\
\hline 8 & 22 & -0.001033058 & -0.001033058 \\
\hline 9 & 23 & -0.000945180 & -0.000945180 \\
\hline 10 & 24 & -0.000868056 & -0.000868056 \\
\hline 11 & 25 & -0.000800000 & -0.000800000 \\
\hline 12 & 26 & -0.000739645 & -0.000739645 \\
\hline 13 & 27 & -0.000685871 & -0.000685871 \\
\hline 14 & 28 & -0.000637755 & -0.000637755 \\
\hline 15 & 29 & -0.000594530 & -0.000594530 \\
\hline 16 & 30 & -0.000555556 & -0.000555556 \\
\hline 17 & 31 & -0.000520291 & -0.000520291 \\
\hline 18 & 32 & -0.000488265 & -0.000488281 \\
\hline 19 & 33 & -0.000458659 & -0.000459137 \\
\hline 20 & 34 & -0.000428251 & -0.000432526 \\
\hline 21 & 35 & -0.000391716 & -0.000408163 \\
\hline 22 & 36 & -0.000346009 & -0.000385802 \\
\hline 23 & 37 & -0.000289506 & -0.000365230 \\
\hline 24 & 38 & -0.000220203 & -0.000346260 \\
\hline 25 & 39 & -0.000135248 & -0.000328731 \\
\hline 26 & 40 & -0.000030638 & -0.000312500 \\
\hline 27 & 41 & 0.000099234 & \\
\hline 28 & 42 & 0.000262330 & \\
\hline 29 & 43 & 0.000470191 & \\
\hline 30 & 44 & 0.000740006 & \\
\hline 31 & 45 & 0.001098237 & \\
\hline 32 & 46 & 0.001587306 & \\
\hline 33 & 47 & 0.002278652 & \\
\hline 34 & 48 & 0.003300210 & \\
\hline 35 & 49 & 0.004899700 & \\
\hline 36 & 50 & 0.007608816 & \\
\hline 37 & 51 & 0.012742933 & \\
\hline 38 & 52 & 0.024318257 & \\
\hline 39 & 53 & 0.059761142 & \\
\hline 40 & 54 & 0.280510990 & \\
\hline
\end{tabular}

${ }^{a}$ Sturmian basis functions are used with the parameter $\alpha$ [Eq. (2)] adjusted in such a way that the Rydberg levels of central interest are approximately $n=$ 25. The positive energy states describe the continuum. Atomic units are used.

simplicity of the Hamiltonian matrix elements. Thus, for example, for the unperturbed hydrogenic Hamiltonian, Eq. (3), the matrix elements have the following form:

$$
H_{n^{\prime} l^{\prime}, n l}^{(0)}=[(\alpha n-1) / 2] \delta_{n n^{\prime}} \delta_{l l^{\prime}}+\left(E_{0} / 2\right) \Delta_{n^{\prime} l^{\prime}, n l} \delta_{l l^{\prime}},
$$

where $\Delta$ 's are the overlap matrix elements

$$
\Delta_{n^{\prime} l^{\prime}, n l}=\int_{0}^{\infty} S_{n^{\prime} l^{\prime}}(r) S_{n l}(r) \mathrm{d} r .
$$

The results shown in Table 2 are obtained by solving the eigenvalue problem

$$
\left[H_{n^{\prime}, r, n l}^{(0)}-E \Delta_{n^{\prime}, n, n}\right] \psi(r)=0 .
$$

To perform Floquet matrix calculations, one also needs the dipole coupling matrix elements $\left\langle S_{n^{\prime} l^{\prime}}(r)|r| S_{n l}(r)\right\rangle$, which can also be easily evaluated analytically.
Figures 3(a) and 3(b) show the ac Stark shifts of $n=11$ and $n=10$ atomic states, respectively, for $l=0,1,2,3$. Forty Sturmian basis functions are used for each angular momentum block $(l=0,1,2,3,4)$, and five Floquet blocks ( $A, A \pm 2 \omega I, A \pm 4 \omega I$ ) are used in the Floquet eigenvalue analysis. The inverse iteration technique ${ }^{10}$ is used to calculate only those quasi-energy eigenvalues and eigenvectors of interest. (The $10 s$ weak-field results agree within $1 \%$ of the perturbation data of Ref. 8.) Figures 3(a) and 3(b) reveal several essential energy-shift behaviors of excited states: (a) All the excited levels shown are shifted upward and closely follow the shift caused by the ponderomotive potential $\bar{V}$ (shown by dotted curves)

$$
\bar{V}=e^{2} F^{2} / 4 m \omega^{2}
$$

in the weaker-field region. This effective potential $\bar{V}$ has its origin in the $A^{2}$ term (where $A$ is the vector potential) and can be shown to be equal to the average quiver kinetic energy picked up by an electron of mass $m$ and charge $e$ driven
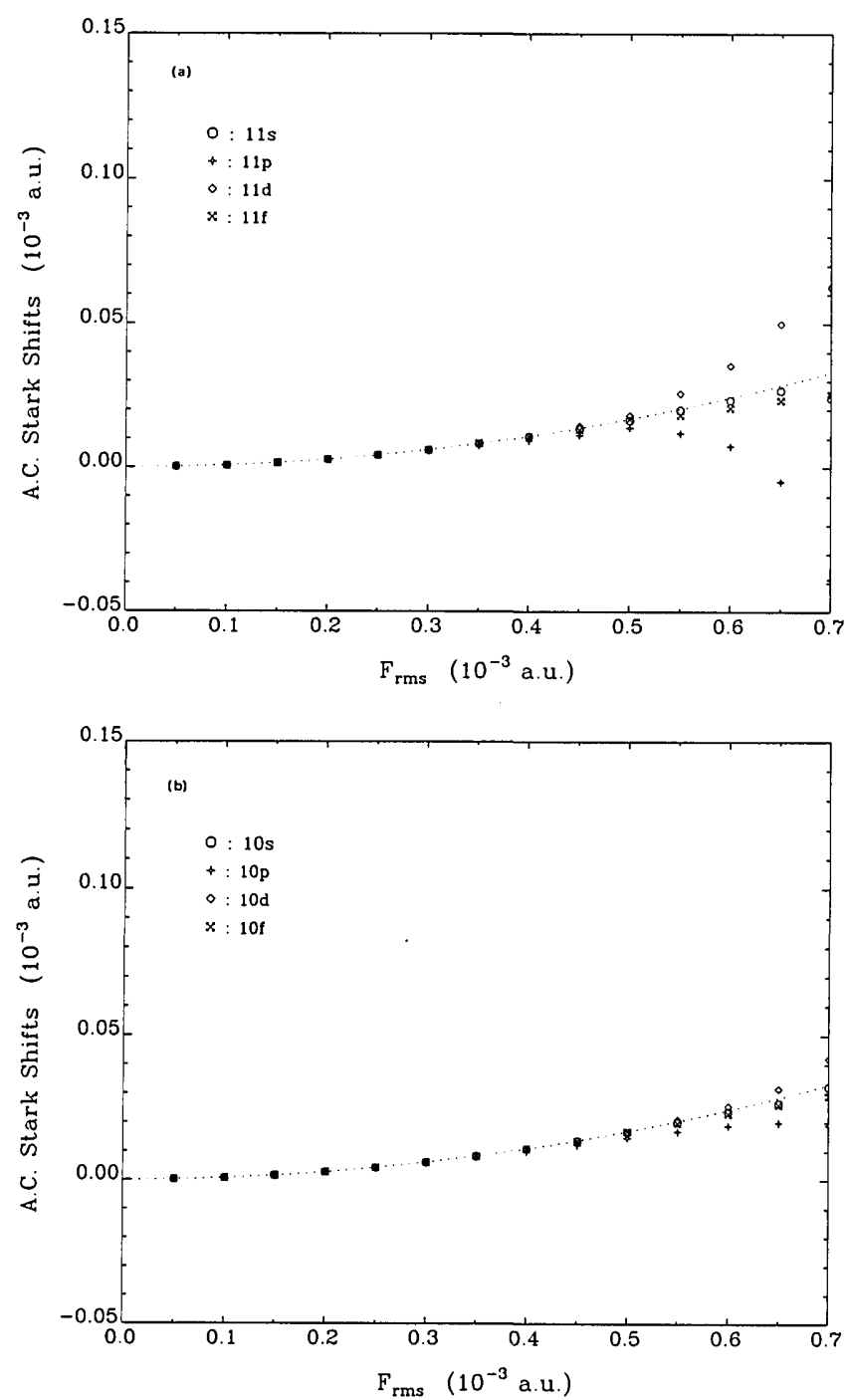

Fig. 3. Alternating current Stark shifts of (a) $n=11$ and (b) $n=10$ atomic states for $l=0,1,2,3$. Level splitting and strong mixings of atomic states occur in higher fields. The dotted curves shown are the ponderomotive potential quadratic shifts $e^{2} F^{2} / 4 m \omega^{2}$. 


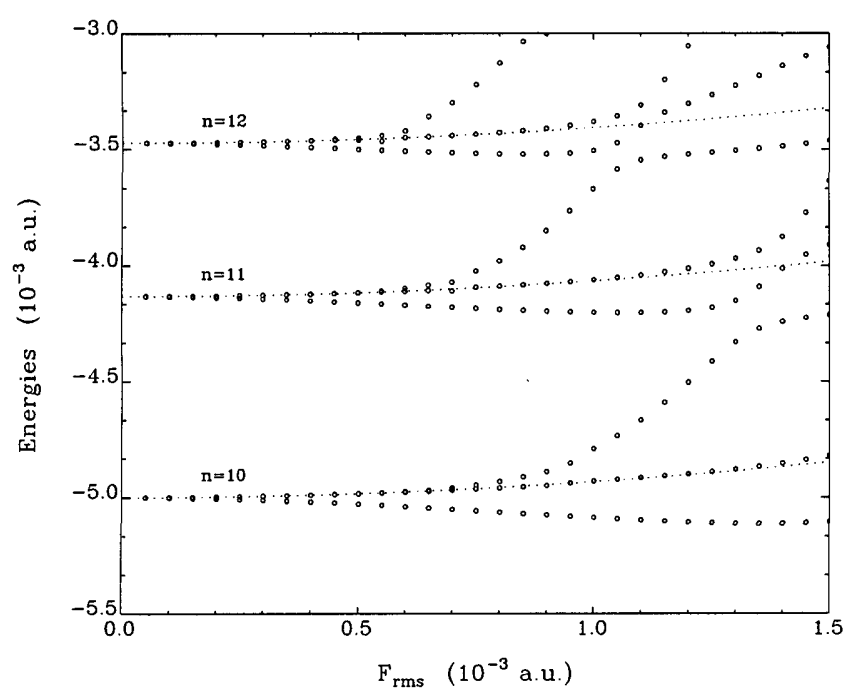

Fig. 4. Intensity-dependent energy-level-shift pattern for evenparity $(s, d, g)$ states for $n=12,11,10$. Notice the large deviation from the ponderomotive potential curves (dotted) in stronger-field regions.

sinusoidally by the fields. Our results lend further support to the view ${ }^{22}$ that all Rydberg states and the continuum are upshifted by the same amount, described by $\bar{V}$. However, this description appears valid only in the weak-field regime where no strong mixings exist among atomic states. (b) Above some critical field strengths $\left(F_{c}\right)$, the atomic energy levels (for a given $n$ but different $l$ ) split, and significant deviation from the $A^{2}$ curve occurs. The critical field strength $F_{c}$ depends on $n$ and decreases rather rapidly as $n$ increases, as can be seen from these figures. One should therefore use the $A^{2}$ shift law with caution in the interpretation of energy-level shifts in high-intensity MPI/ATI experiments. (c) For $F>F_{c}$, strong mixings exist among nearby atomic states, and the level identities usually cannot be discerned. [The level (symbol) assignment in Figs. 3(a) and $3(\mathrm{~b})$ is therefore applicable only to weaker-field regions.]

To see the intricate and global level-shift behavior in strong fields, in Fig. 4 we show the even-parity $(s, d, g)$ state quasi-energies for $n=12,11,10$. (The odd-parity ( $p$ and $f$ ) states show up in a different quasi-energy regime and are separated for clarity. They are, of course, mixed strongly with the even-parity states in strong fields). For each $n$, the upper branch has the dominant $d$ characteristic, the middle branch has the dominant $s$ characteristic, while the lower branch has the dominant $g$ characteristic. (The $g$ states are less converged because our basis set includes only $l=0,1,2,3,4$ Sturmian functions.) Above some field strengths, mixings among nearby $n=$ manifolds can take place, and avoided-crossing patterns appear. All quasi-energy levels lose their identities, and no atomic level assignment is possible in these strong regions. In Fig. 4, the dotted curves again indicate how the atomic energy levels (for each $n$ ) would behave if they follow the ponderomotive potential shift. Large deviation from the $A^{2}$ curve is clearly evident in the stronger-field regime. (Interestingly, except in the avoided-crossing regions, the $s$-characteristic dominant states (middle branches) rather closely follow the $A^{2}$ curves.)

Figure 5 shows the intensity-dependent energy-level shift pattern for highly excited states ( $n=49,50,51,52$ ). (For clarity, only the even-parity $(s, d, g)$ states are shown, as in Fig. 4.) Eighty Sturmian basis functions are used for each angular momentum block in this calculation. The levelshift pattern is similar to that shown in Fig. 4, except that the critical field strengths $F_{c}$ are now considerably lower. Also, the less-perturbed g-states (the lowest branch for each $n$ ) now closely follow the ponderomotive potential $\left(A^{2}\right)$ curves (dotted curves). For $F>F_{c}$, a large departure from the $A^{2}$ shift occurs for the upper and middle branch states (for each $n$ ), and strong inter- $n$ mixings take place. This behavior is expected to prevail for all Rydberg levels.

\section{CLASSICAL TREATMENT OF MULTIPLE- HARMONIC GENERATION ACCOMPANYING MULTIPHOTON IONIZATION}

Recently high-order (up to order 33) harmonic generation has been observed when $\mathrm{Xe}, \mathrm{Kr}$, or $\mathrm{Ar}$ atoms are irradiated by intense $\left(10^{13}-10^{14} \mathrm{~W} / \mathrm{cm}^{2}\right)$ pulsed laser fields. ${ }^{11-13}$ Several unexpected features have been revealed. For example, the experiments by Li et al. ${ }^{13}$ show a strong third harmonic followed by a succession of odd harmonics (up to 33rd order in Ar). While the decrement from third to fifth harmonic is steep (about 2 orders of magnitude), the relative intensities of higher harmonics (7th-27th harmonics) are roughly equal in strength and fall off rather slowly. After the 27th harmonic, the intensities drop significantly and show a cutoff at the 33rd harmonic. A continuous background of scattered light runs through all the harmonics.

In a previous paper ${ }^{4}$ we showed that classical trajectory treatment of the electronic motion in intense laser fields can offer detailed insights regarding the MPI/ATI mechanisms as well as the continuum threshold shift phenomenon. In this section we extend this research and show that a spectral analysis of the classical trajectories can reveal the mechanism responsible for the high-order harmonic generation in a straightforward manner. In addition, our results offer a

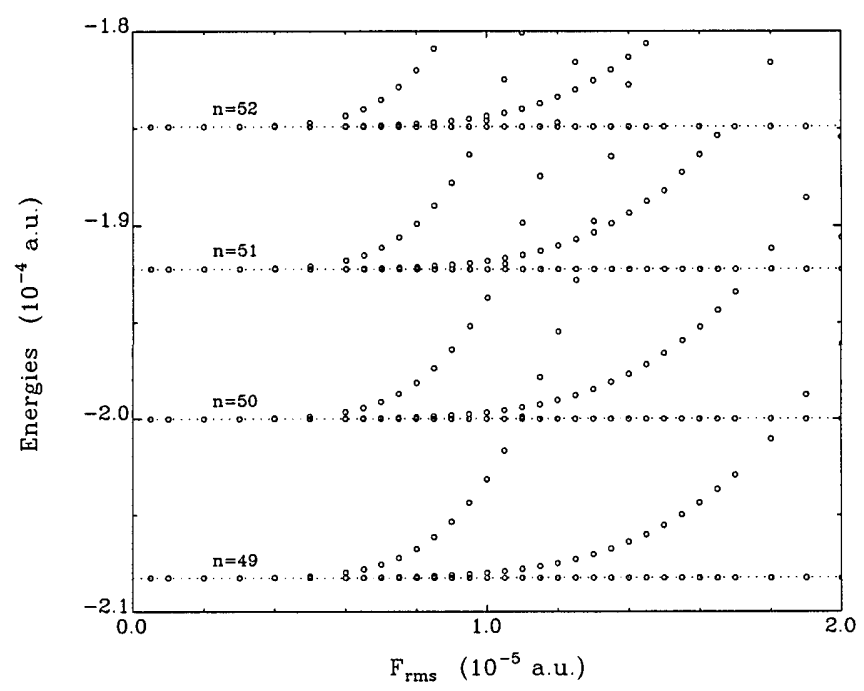

Fig. 5. Intensity-dependent energy-level-shift pattern for evenparity $(s, d, g)$ states for highly excited states $n=49,50,51,52$. Ponderomotive curves are shown (dotted). 

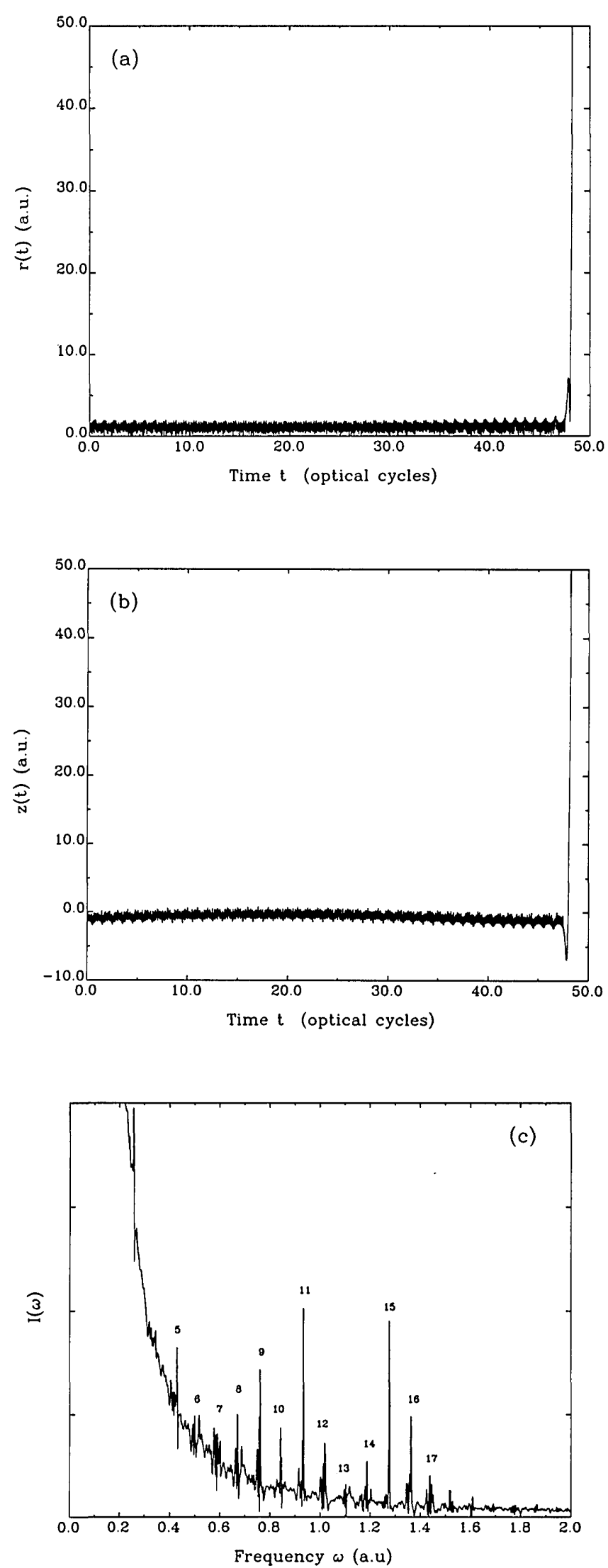

Fig. 6. (a) Electron radius trajectory $r(t)$ and (b) dipole moment function $z(t)$ as a function of time (measured in number of field optical cycles) for a direct ionization classical trajectory. (c) Power spectrum $I(\omega)$ of $z(t)$. The physical parameters used are $\lambda=530 \mathrm{~nm}$ and $F=0.15$ a.u. possible explanation of the continuous background observed in the experiments.

The classical Hamiltonian function is derived from the quantum-mechanical Hamiltonian given in Eq. (1). The effect of the manner in which fields are turning on and off is not considered here but will be treated in a subsequent publication. Hamilton's equations of motion for the electron are solved numerically, using the variable-order, variable-step Adams method. ${ }^{23}$ The initial conditions for the motion of the electron at $t=0$ are chosen by standard Monte Carlo methods from the microcanonical distribution with energy $E=-0.5$ a.u. to simulate the statistical distribution characteristic of the initial physical conditions of the classical $\mathrm{H}$-atom ground state. ${ }^{4}$

We consider the power spectrum or spectral density of the dipole moment function $z(t)$,

$$
I(\omega)=(1 / 2 \pi) \lim _{T \rightarrow \infty}(1 / 2 T)\left\langle\left|\int_{0}^{2 T} z(t) \exp (-i \omega t) \mathrm{d} t\right|^{2}\right\rangle
$$

where \langle\rangle indicates an average over an ensemble appropriate to the problem. In practice, a single trajectory is sufficient to illustrate the underlying mechanisms. In the absence of the Coulomb field, the electron (in an oscillating field $e F_{z}$ $\cos \omega t$ ) moves uniformly on average, but superimposed on this uniform motion is a sinusoidal oscillation with characteristic velocity given by

$$
\mathbf{v}=\mathbf{v}_{0}+(e F / m \omega) \hat{z} \sin \omega_{L} t .
$$

In the presence of both the Coulomb and the oscillating electric fields, the electron can gain or lose energy and angular momentum and undergoes MPI/ATI, as we have shown in a previous paper. ${ }^{4}$

There are two types of classical trajectory leading to ionization of electrons: (a) direct excitation of the atom from the ground state to the individual continuum by nonresonant multiphoton absorption and (b) sequential excitation, in which an electron is first multiphoton-excited to a Rydberg orbit and then further excited to the continuum by absorption of additional photon(s).

Figures 6(a), 6(b), and 6(c) show, respectively, a typical direct ionization trajectory $r(t)$, the dipole moment function $e z(t)(e=1)$, and its corresponding power spectrum $I(\omega)$. The physical parameters used are $F=0.15$ a.u. and $\lambda=530$ $\mathrm{nm}$. After oscillating around the nucleus for about 48 optical cycles, the electron is ejected directly to the continuum. The power spectrum shows that a series of equally spaced peaks sit at approximately $N \omega_{L}$ positions, where $N$ 's are integer numbers and $\omega_{L}$ is the frequency of the laser. The dominant peaks occur at $N=9, N=11$, and $N=15$, but most of the peaks are roughly the same order of intensity. ${ }^{24}$ After the $N=17$ peak, the intensity drops significantly. This phenomenon is similar to the high-order harmonic generation observed in strong field MPI/ATI experiments. ${ }^{11-13}$ There is a giant peak in the lower-frequency region, and a continuous background runs through all the harmonic peaks. We found that these features are attributed to the ionizing portion of the trajectory. The magnitude of the giant peak is sensitive to the cutoff time of the trajectory, as expected. The high-order harmonic generation peaks are entirely due to the fast-oscillating portion of the trajectory, i.e., the portion before ionization. 

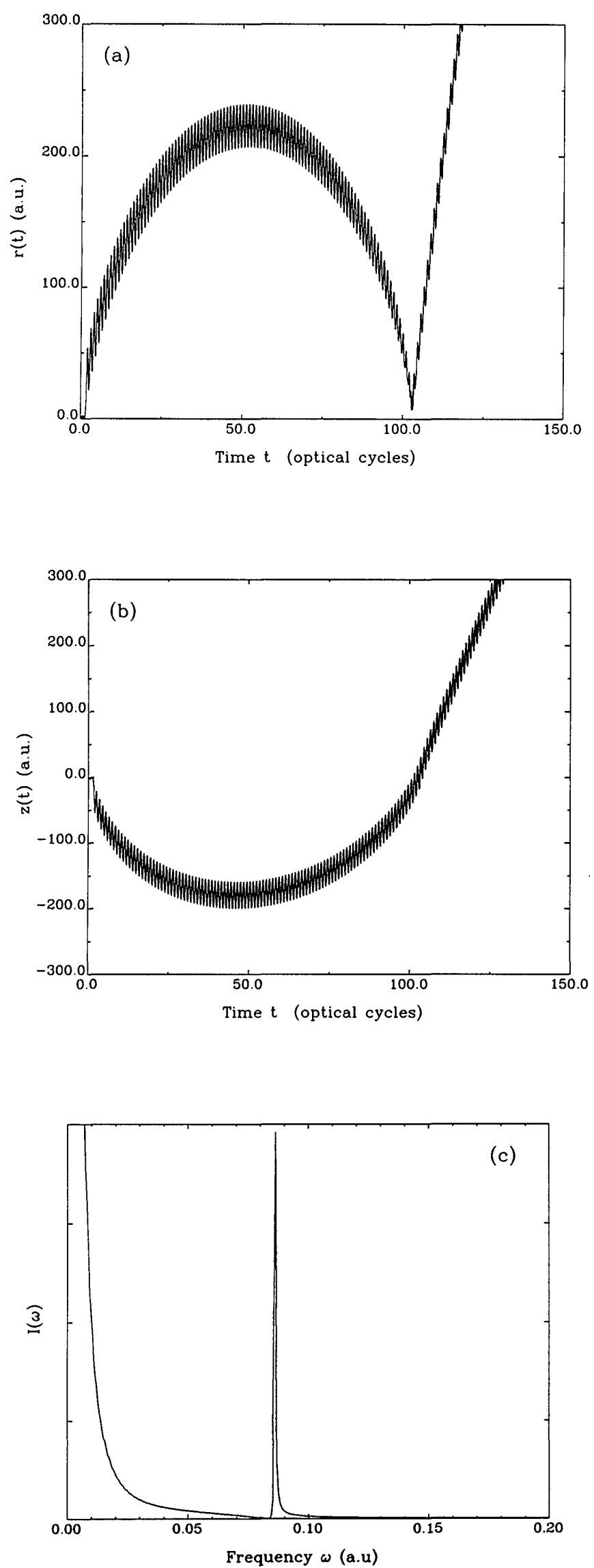

Fig. 7. (a) $r(t)$, (b) $z(t)$, and (c) $I(\omega)$ for a sequential-excitation-type trajectory. Parameters as in Fig. 6.
Figure 7(a) shows a typical sequential excitation trajectory in which the electron is first pumped by the external fields to a Rydberg state. This corresponds to an electron's moving in a large Kepler orbit with wobbles caused by the oscillating field. After moving around the orbit for about 100 optical cycles, the electron reaches the perihelion point close to the nucleus, absorbs additional photon(s), and is suddenly ionized. The dipole moment function $z(t)$ and its power spectrum $I(\omega)$ are shown, respectively, in Figures 7(b) and 7(c). Interestingly, while the motion of the electron appears more spectacular in this case, its power spectrum consists of only a single peak precisely located at the laser frequency $\omega_{L}=(0.08596$ a.u. or $530 \mathrm{~nm})$. (The lower-frequency larger peak is again due to the ionizing portion of the trajectory.) This single-frequency peak at $\omega_{L}$ is clearly due to the wobble of $z(t)$ induced by the oscillating field. We see no higher-order harmonic components in this type of trajectory.

It is perhaps not surprising to find that only the direct excitation type (Fig. 6) of trajectory is responsible for the observed high-order harmonic generation. Only when the electron is close to the nucleus can the electron exchange energy and angular momentum efficiently with the electromagnetic fields. Quantum mechanically, this implies that the initially bound-state electronic wave function builds up overlapping components with the individual continuum in a direct fashion while the electron is still in the vicinity of the nucleus. This is the basic mechanism responsible for highintensity MPI/ATI and high-order harmonic generation. Our results also suggest that the continuous background runs through the high-order harmonic peaks observed in the experiments ${ }^{11-13}$ and in quantum model calculations ${ }^{14,15}$ may be attributed to the ionizing portion of the trajectory (wave function).

In conclusion, the spectral analysis of classical trajectories offers a powerful complementary tool to quantum-mechanical methods for the exploration of physical mechanisms in MPI/ATI processes and multiple harmonic generation in strong fields. This classical spectral technique may be made more quantitative by means of appropriate semiclassical quantization of the classical trajectories. Research in this direction is in progress.

\section{ACKNOWLEDGMENT}

This work was supported in part by the Department of Energy (Division of Chemical Sciences) and University of Kansas General Research Funds.

* Current address, Department of Chemistry, California Institute of Technology, Pasadena, California.

\section{REFERENCES AND NOTES}

1. R. R. Freeman, P. H. Bucksbaum, H. Milchberg, S. Darrack, D. Schumacher, and M. E. Geusic, Phys. Rev. Lett. 59, 1092 (1987).

2. P. Agostini, A. Antonetti, P. Breger, M. Crance, A. Migus, H. G. Muller, and G. Petite, J. Phys. B. 22, 1971 (1989).

3. S. I. Chu and J. Cooper, Phys. Rev. A 32, 2769 (1985).

4. S. I. Chu and R. Y. Yin, J. Opt. Soc. Am. B 4, 720 (1987).

5. R. Shakeshaft and X. Tang, Phys. Rev. A 36, 3193 (1987).

6. See, for example, L. Pan, K. T. Taylor, and C. W. Clark, Phys. Rev. Lett. 61, 2673 (1988), and references therein. 
7. R. Kulander, Phys. Rev. A 35, 445 (1987).

8. See, for example, E. Arnous, J. Bastian, and A. Maquet, Phys. Rev. A 27, 977 (1983), and references therein.

9. Y. Gontier and M. Trahin, Phys. Rev. A 40, 1351 (1989).

10. S. I. Chu and W. P. Reinhardt, Phys. Rev. Lett. 39, 1195 (1977); A. Marquet, S. I. Chu, and W. P. Reinhardt, Phys. Rev. A 27, 2946 (1983).

11. M. Ferray, A. L'Huillier, X. F. Li, L. A. Lompré, G. Mainfray, and C. Manus, J. Phys. B 21, L31 (1988).

12. A. McPherson, G. Gibson, H. Jara, U. Johann, T. S. Luk, I. A. McIntyre, K. Boyer, and C. K. Rhodes, J. Opt. Soc. Am. B 4, 595 (1987).

13. X. F. Li, A. L'Huillier, M. Ferray, L. A. Lompré, and G. Mainfray, Phys. Rev. A 39, 5751 (1989).

14. K. C. Kulander and B. W. Shore, Phys. Rev. Lett. 62, 524 (1989).

15. J. H. Eberly, Q. Su, and J. Javanainen, Phys. Rev. Lett. 62, 881 (1989).

16. S. I. Chu, Adv. At. Mol. Phys. 21, 197 (1985).

17. J. H. Shirley, Phys. Rev. 138, B979 (1965).

18. For a recent review on various generalizations of Floquet theories and techniques for the treatment of intense-field multipho- ton and nonlinear optical processes, see S. I. Chu, Adv. Chem. Phys. 73, 739 (1989).

19. B. R. Junker, Adv. At. Mol. Phys. 18, 207 (1982); W. P. Reinhardt, Annu. Rev. Phys. Chem. 33, 223 (1982).

20. M. Rotenberg, Adv. At. Mol. Phys. 6, 233 (1970).

21. For other recent treatments of MPI/ATI processes using the Sturmian basis, see, for example, E. Karule, J. Phys. B 21, 1997 (1988); R. M. Potvliege and R. Shakeshaft, Phys. Rev. A 38, 1098 (1988).

22. P. Avan, C. Cohen-Tannoudji, J. Dupont-Roc, and C. Fabre, J. Phys. (Paris) 37, 993 (1976); S. Liberman, J. Pinard, and A. Taleb, Phys. Rev. Lett. 50, 888 (1983); L. Hollberg and J. L. Hall, Phys. Rev. Lett. 53, 230 (1984).

23. G. Hall and J. M. Watt, eds. Modern Numerical Methods for Ordinary Differential Equations (Clarendon, Oxford, 1976).

24. Even-order harmonic peaks are also observed, though smaller in magnitude in general. This may be attributed to the nature of the classical treatment, which allows a continuous range of orbital angular momentum values. By running a swarm of trajectories with appropriate initial conditions, the even-order harmonic peaks can be quenched. 